\title{
Fibroblast Growth Factor 23 Genotype and Cardiovascular Disease in Patients Undergoing Hemodialysis
}

\author{
Tae-Hwi Schwantes-An ${ }^{a}$ Sai Liu ${ }^{b}$ Margaret Stedman ${ }^{b}$ Brian S. Decker ${ }^{c}$ \\ Leah Wetherill $^{\mathrm{a}}$ Howard J. Edenberg ${ }^{\mathrm{a}, \mathrm{d}}$ Matteo Vatta ${ }^{\mathrm{a}}$ Tatiana M. Foroud $^{\mathrm{a}}$ \\ Glenn M. Chertow ${ }^{b}$ Sharon M. Moe ${ }^{c, e}$ \\ ${ }^{a}$ Department of Medical and Molecular Genetics, Indiana University School of Medicine, Indianapolis, IN, USA; \\ ${ }^{b}$ Division of Nephrology, Department of Medicine, Stanford University School of Medicine, Stanford, CA, USA; \\ 'Division of Nephrology, Department of Medicine, Indiana University School of Medicine, Indianapolis, IN, USA; \\ ${ }^{\mathrm{d}}$ Department of Biochemistry and Molecular Biology, Indiana University School of Medicine, Indianapolis, IN, USA; \\ e Roudebush Veterans Administration Medical Center, Indianapolis, IN, USA
}

\section{Keywords}

Dialysis · Cardiovascular disease $\cdot$ Mortality $\cdot$ Fibroblast growth factor-23 . Chronic kidney disease-mineral bone disorder

\begin{abstract}
Background: Elevated serum concentrations of fibroblast growth factor 23 (FGF23) are associated with cardiovascular mortality in patients with chronic kidney disease and those undergoing dialysis. Objectives: We tested the hypotheses that polymorphisms in FGF23, its co-receptor alpha-klotho $(K L)$, and/or FGF23 receptors (FGFR) are associated with cardiovascular events and/or mortality. Methods: We used 1,494 DNA samples collected at baseline from the Evaluation of Cinacalcet $\mathrm{HCl}$ Therapy to Lower Cardiovascular Events Trial, in which patients were randomized to the calcimimetic cinacalcet or placebo for the
\end{abstract}

treatment of secondary hyperparathyroidism. We analyzed European and African Ancestry samples separately and then combined summary statistics to perform a meta-analysis. We evaluated single-nucleotide polymorphisms (SNPs) in FGF23, KL, and FGFR4 as the key exposures of interest in proportional hazards (Cox) regression models using adjudicated endpoints (all-cause and cardiovascular mortality, sudden cardiac death, and heart failure [HF]) as the outcomes of interest. Results: rs11063112 in FGF23 was associated with cardiovascular mortality (risk allele $=A$, hazard ratio $[H R] 1.32$, meta- $p$ value $=0.004)$ and HF (HR 1.40, meta- $p$ value $=0.007$ ). No statistically significant associations were observed between FGF23 rs13312789 and SNPs in FGFR4 or $K L$ genes and the outcomes of interest. Conclusions: rs11063112 was associated with HF and cardiovascular mortality in patients receiving dialysis with moderate to severe secondary hyperparathyroidism.

(C) 2019 S. Karger AG, Base

\section{KARGER}

(c) 2019 S. Karger AG, Basel

E-Mail karger@karger.com

www.karger.com/ajn
Sharon M. Moe, MD

Division of Nephrology, Stuart A. Kleit Professor of Medicine, Professor of Anatomy and Cell Biology, Director, Indiana University School of Medicine 950 W. Walnut Street, R2-202, Indianapolis, IN 46202 (USA)

E-Mailsmoe@iu.edu 


\section{Introduction}

Chronic kidney disease-mineral bone disorder (CKD-MBD) is a systemic syndrome characterized by derangements in mineral metabolism, extra osseous calcification, and bone fragility [1]. Fibroblast growth factor 23 (FGF23) is produced in bone osteoblasts, with a primary effect on renal tubules through FGF receptor 1 (FGFR1) to increase phosphate excretion and enhance synthesis of $1,25(\mathrm{OH})_{2}$-vitamin $\mathrm{D}$ in conjunction with its co-receptor a-klotho (KL). In the heart, FGF23 binds to FGFR4 in a KL-independent manner and directly induces left ventricular hypertrophy (LVH) in animal models $[2,3]$. Serum concentrations of FGF23 become markedly elevated in patients with $\mathrm{CKD}$ and are associated with progression of LVH $[4,5]$ and mortality [6, 7]. Cinacalcet-induced reduction in serum FGF23 was associated with a lower risk of cardiovascular death, adjudicated heart failure (HF) events, and sudden cardiac death (SCD) [7]. These data suggest that DNA polymorphisms in the genes encoding FGF23 (FGF23), FGFR4 (FGFR4), or a-KL may contribute to cardiovascular disease in patients with CKD.

To explore the potential role of genetic polymorphisms in the FGF23-KL pathway on cardiovascular disease in patients receiving hemodialysis, we utilized DNA samples from the Evaluation of Cinacalcet $\mathrm{HCl}$ Therapy to Lower Cardiovascular Events (EVOLVE) Trial $[8,9]$. In EVOLVE, 3,883 patients receiving hemodialysis with moderate to severe secondary hyperparathyroidism (sHPT; baseline parathyroid hormone (PTH) $\geq 600 \mathrm{pg} /$ $\mathrm{mL}$, and serum calcium $\geq 8.4 \mathrm{mg} / \mathrm{dL}$ ) were randomized to receive either cinacalcet or placebo, in addition to standard of care treatments that generally included phosphate binders and calcitriol or vitamin D analogs. Mortality and cardiovascular events were assessed and adjudicated by an independent Clinical Events Committee. Patients were followed for up to 64 months, and DNA was collected in a subset of these subjects. The present study sought to determine if known single-nucleotide polymorphisms (SNPs) in FGF23, FGFR4, or KL are associated with mortality and/or cardiovascular events.

\section{Materials and Methods}

DNA was collected at baseline from those patients in the EVOLVE trial who signed a separate consent $(n=1,919)$. EVOLVE was an international study in 22 countries, and not all countries allowed the collection of DNA for research purposes. Blood samples were processed by the central study laboratory (Covance,
Princeton NJ), and deidentified samples sent to Indiana University. The study was deemed not subject to the Common Rule by the Indiana University Institutional Review Board. After DNA extraction, 1,852 samples were genotyped using the Sequenom Mass-Array system. DNA and genotyping quality assessment have been described previously [10]. We categorized ancestry group by self-reported race. For this report, we only included patients of European Ancestry (EA; $n=1,083)$ and African Ancestry (AfAn, $n=411$ ) due to small numbers of patients in other selfreported race groups (i.e., Asians, Aboriginal and other Native persons, and persons of mixed race). Based on previously published literature reporting an association of SNPs in genes in the FGF23-KL pathway, we genotyped 5 SNPs in FGFR4, 2 SNPs in FGF23, and 2 SNPs in KL [11-15]. There was no significant deviation from Hardy-Weinberg equilibrium for any SNP in either ancestry group (all $p>0.21$ ).

\section{Statistical Analyses}

Descriptive statistics to characterize the groups are provided in Table 1. As primary analysis, association between 9 SNPs and 4 EVOLVE outcomes relevant to the known biologic effects of FGF23 on the heart (all-cause mortality, cardiovascular mortality, SCD, and HF) were tested using proportional hazards regression (Cox) models, or the Fine-Gray modification of the Cox model when accounting for competing risks (i.e., noncardiovascular death). All models were stratified by site and presence of diabetes. Study participants were followed up for up to 64 months. We included age, sex, vintage (number of years since starting dialysis), history of smoking (current or past), and treatment assignment (placebo/cinacalcet in addition to standard treatment) as covariates [8]. A global test combining zero-slope tests on the scaled Schoenfeld residuals plotted against time was used to examine the proportional hazards assumption for each covariate in the model. We considered death as a competing risk in the evaluation of nonfatal events. For the analyses including competing risks, we constructed cumulative incidence rather than survival plots for each genotype and calculated subdistribution hazards.

We utilized an additive genetic model, which assumed a similar increase (or decrease) in the hazard ratio (HR) for each copy of the coded allele. Our primary analysis of interest was a metaanalysis across the 2 ancestry groups, EA and AfAn, in order to account for the known differences in cardiovascular risk $[16,17]$ and minor allele frequencies. To compare results across groups in the meta-analysis, we assigned the minor alleles of the EA group as the reference alleles in both EA and AfAn samples. All meta-analysis heterogeneity $p$-values were greater than 0.06 , suggesting there was no significant heterogeneity between the 2 population groups, and meta-analysis of the EA and AfAn summary statistics was performed using R package "r-meta" (https://cran. r-project.org/web/packages/rmeta/index.html, 2.16). The inverse SEs were implemented as weights. We accounted for sample heterogeneity by using a random effects model. We performed all analyses using SAS 9.4 (SAS Institution Inc, Cary, NC, USA) and R.

Due to the correlation among the outcomes, and the correlation among the SNPs, the number of independent tests in this study was not simply the product of the number of outcomes times the number of SNPs. The Matrix Spectral Decomposition method (MatSpD, https://gump.qimr.edu.au/general/daleN/ matSpD/) was used to estimate the number of independent tests, 
Table 1. Demographic and baseline laboratory values for DNA cohort compared to the primary EVOLVE trial

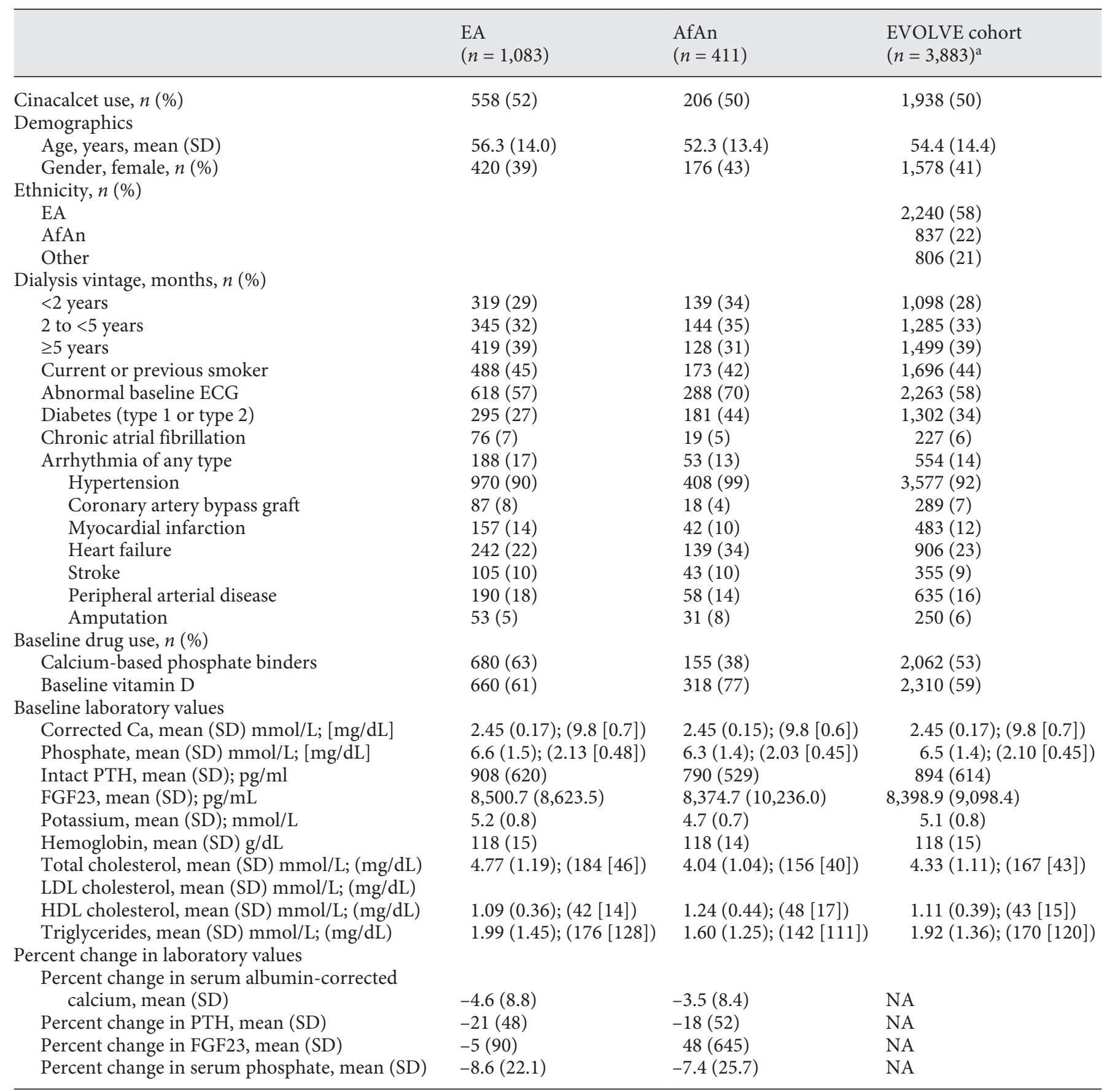

${ }^{\text {a }}$ From references [7-9, 17].

EVOLVE, Evaluation of Cinacalcet $\mathrm{HCl}$ Therapy to Lower Cardiovascular Events; FGF23, fibroblast growth factor 23; EA, European Ancestry; AfAn, African Ancestry; NA, not applicable. 


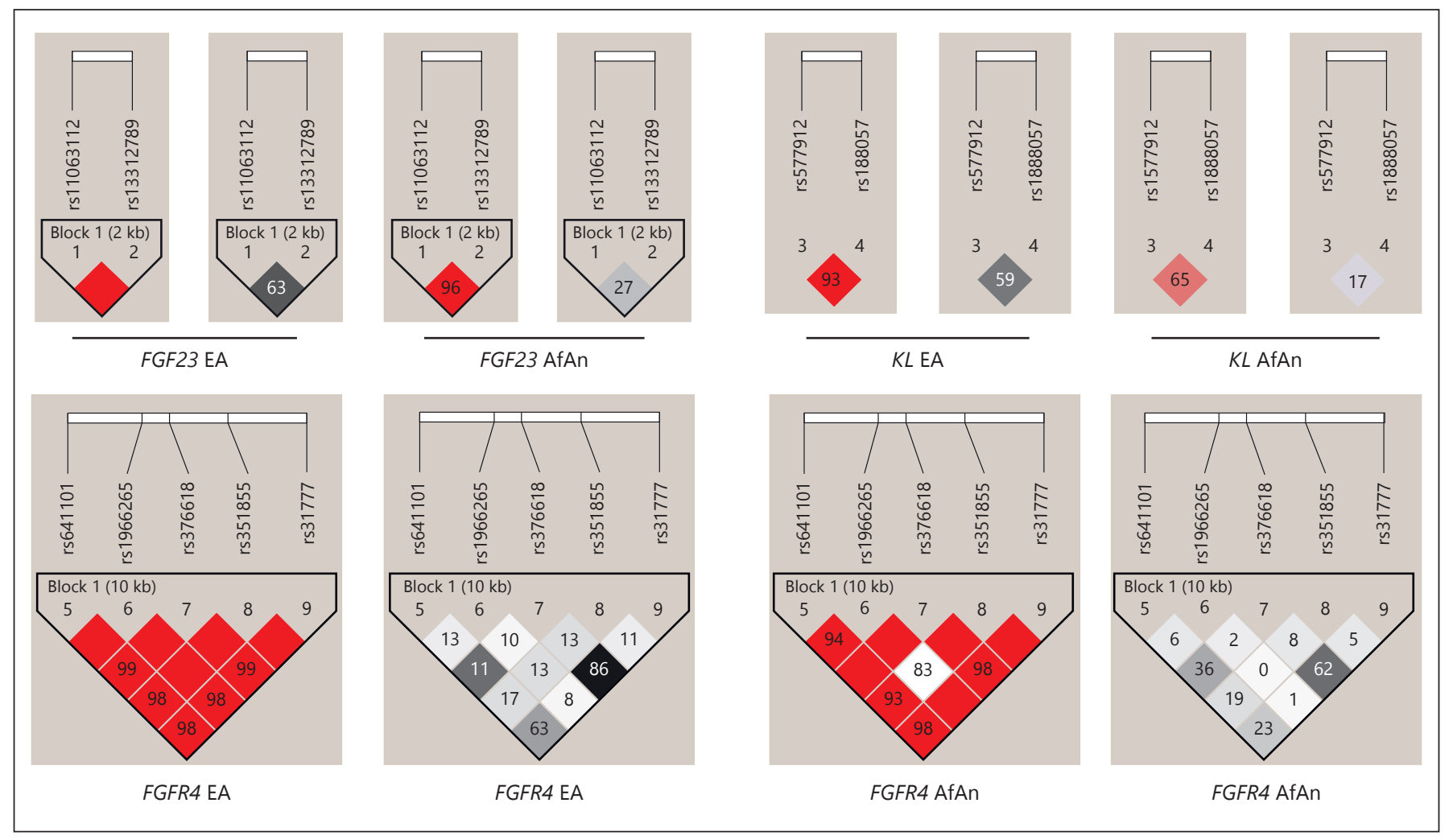

Fig. 1. Linkage disequilibrium (LD) in FGF23, KL, and FGFR4: LD plots for the FGF23, KL, and FGFR4 SNPs examined in the European Ancestry (EA) and the African Ancestry (AfAn) samples. For

each gene and ancestry combination, left panel shows LD measured in $\mathrm{D}^{\prime}$, and right panel shows LD measured in $r^{2}$. FGF23, fibroblast growth factor 23; KL, alpha-klotho; FGFR4, FGF receptor 4.

based on the correlation matrix of the outcomes (online suppl. Table 1; for all online suppl. material, see www.karger.com/ doi/10.1159/000496060) and on the correlation matrix of the SNPs (D'values of LD; Fig. 1). The MatSpD method was applied separately in the EA and AfAn samples. As the SNPs had lower linkage disequilibrium in the AfAn individuals, this sample was estimated to have a larger number of independent tests compared with the EA sample. Hence, the AfAn MaTSpD results included 6.21 independent tests ( $=1.6$ independent outcomes $x 3.88$ independent SNPs), and this factor was used to correct for multiple testing for both populations. This corresponded to a corrected alpha threshold of 0.008 . To aid the interpretation of survival analysis results, secondary analyses tested for association between the 9 SNPs and the following biochemical measurements: baseline serum calcium, phosphate, PTH, $1.25(\mathrm{OH})_{2}$-vitamin $\mathrm{D}$, and FGF23, along with percentage change in serum concentrations between baseline and 20 week follow-up. Due to nonnormal distributions of the traits, which can inflate type I error rates, we adjusted all baseline and percentage change in serum concentrations for covariates reported in a previous publication [10] and using the same method, obtained residuals and tested associations with the SNPs. As with survival analyses, all analyses linking SNPs to laboratory tests were conducted separately in EA and AfAn samples followed by meta-analysis using summary statistics from the 2 groups.

\section{Results}

Baseline demographic, cardiovascular, and CKDMBD laboratory data for individuals genotyped for at least one of the SNPs are provided in Table 1. A total of 1,494 patients were analyzed (1,083 EA and 411 AfAn samples). Patients who were analyzed in this cohort were, in general, similar to the overall EVOLVE cohort with multiple risk factors for cardiovascular disease (Table 1). Alleles and minor allele frequency for the analyzed SNPs in FGF23, FGFR4, and KL are shown in online supplemental Table 2 and the $\mathrm{LD}$ plots in Figure 1.

Rs1 1063112 was associated with cardiovascular mortality $(\mathrm{HR}=1.32$, meta- $p$ value $=0.004$, A allele increased risk $)$ and $\mathrm{HF}(\mathrm{HR}=1.40$, meta- $p$ value $=0.007$, A allele increased risk; Table 2 and Fig. 2). No significant associations were observed between the other SNPs in FGF23, FGFR4, or KL genes and the 4 tested cardiovascular/mortality outcomes (online suppl. Table 3). 


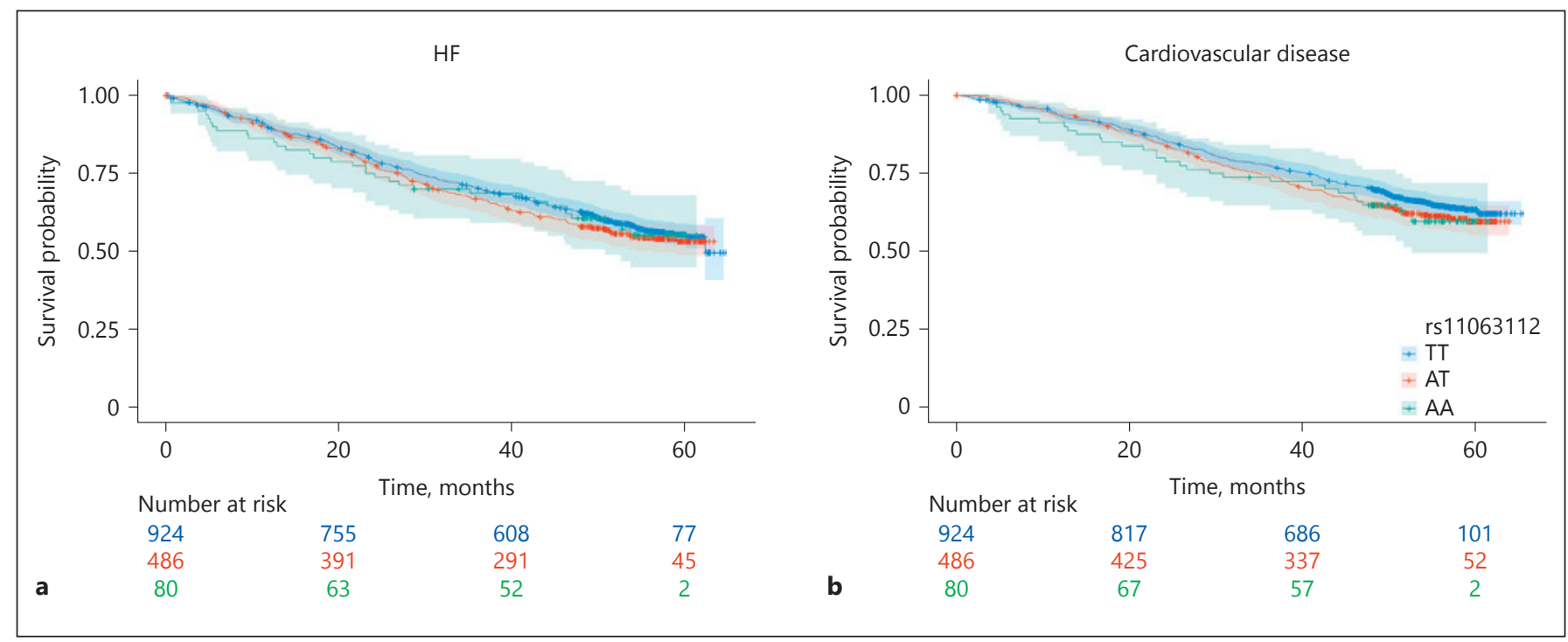

Fig. 2. Probability of survival and SNPs in the FGF23 gene: Unadjusted Kaplan-Meier Curve for survival, or being free from the endpoints in Table 2. a Association between the FGF23 SNP rs11063112 and HF. b Association between the FGF23 SNP

rs11063112 and cardiovascular mortality. The color-shaded area represents the 95\% CI. HF, heart failure; EA, European Ancestry; AfAn, African Ancestry.

Table 2. Association of SNPs with cardiovascular outcomes

\begin{tabular}{lllllllll}
\hline End point & Gene rsID & p value & HR (95\% CI) & $\begin{array}{l}\text { EA } \\
\text { HR }\end{array}$ & $\begin{array}{l}\text { AfAn } \\
\text { HR }\end{array}$ & $\begin{array}{l}\text { Event number } \\
\text { (EA/AfAn) }\end{array}$ & $\begin{array}{l}\text { Censored patients } \\
\text { (EA/AfAn) }\end{array}$ & $\begin{array}{l}\text { Competing } \\
\text { events (EA/AfAn) }\end{array}$ \\
\hline $\begin{array}{l}\text { Heart failure } \\
\begin{array}{c}\text { Cardiovascular } \\
\text { mortality }\end{array}\end{array}$ & FGF23 rs11063112 & 0.007 & $1.40(1.10-1.79)$ & 1.37 & 1.51 & $184(100 / 84)$ & $859(639 / 220)$ & $447(340 / 107)$ \\
\hline
\end{tabular}

Meta-analysis results for survival analysis with cardiovascular outcome adjusted for age, sex, duration of dialysis, history (current/past) of smoking, and treatment (placebo/cinacalcet).

SNP, single nucleotide polymorphism; rsID, reference SNP ID number; HR, hazard ratio; EA, European Ancestry; AfAn, African Ancestry. The HR represents the increased risk for each copy of the reference allele.

In order to understand the potential mechanism of our association between the FGF23 SNP and cardiovascular mortality, we determined if rs 11063122 was also associated with the baseline biochemical measures of $\mathrm{CKD}$ MBD. We tested for the association between rs11063122 and serum calcium (corrected for albumin), phosphate, intact PTH, $1,25(\mathrm{OH})_{2}$-vitamin $\mathrm{D}$, and intact FGF23. No statistically significant associations were observed (online suppl. Table 4). We further tested if rs11063112 modified the treatment (cinacalcet or placebo) effect on the percent change of CKD-MBD laboratory values from baseline to 20 weeks. There was no significant effect modification (online suppl. Table 5).

\section{Discussion/Conclusion}

Cardiovascular disease is the leading cause of death in patients with CKD. Among patients with more advanced $C K D$, the predominant cardiovascular diseases are those related to poorly controlled hypertension and LVH, HF, stroke, and SCD $[18,19]$. In patients receiving dialysis, $\mathrm{LVH}$ is present in more than $75 \%$ of patients $[20,21]$. Multiple studies have demonstrated that the highest serum concentrations of FGF23 are associated with LVH and mortality in CKD and in patients receiving dialysis [2, 4, 22-24]. Herein, we report the novel observation that one SNP in FGF23, rs11063112, is associated with adjudicated cardiovascu- 
lar mortality and HF events in participants from the EVOLVE trial.

We previously reported that a $30 \%$ or more reduction in serum intact FGF23 induced by cinacalcet was associated with lower rates of HF, SCD, and cardiovascular mortality [7]. These results are consistent with the known cardiotoxic effects of FGF23. In rodent models, FGF23 induces cardiac hypertrophy mediated by FGFR4 [2, 25, 26]. Genetic ablation of FGFR4 can prevent the induction of LVH by FGF23 in rodent models of CKD and aging [27]. In autopsy studies of children with advanced CKD, the upregulation of FGF23 and FGFR4 expression in heart tissue were associated with fibrosis [24], a known precursor of diastolic dysfunction, HF, and arrhythmia. In addition, FGF23 can induce a spike in intracellular calcium in cardiomyoctes and alter contractility, physiology predisposing to SCD $[27,28]$. Thus, the findings in the present study support the biologic effects of FGF23.

In the current report, we did not find associations among either SNP in FGF23 with serum FGF23 concentrations. A recent report by Robinson-Cohen et al. [29] also reported a lack of association between variants in FGF23 region and serum FGF23 concentrations in patients without CKD. This may indicate that currently tested SNPs in FGF23 do not modulate serum levels of FGF23. Furthermore, serum concentrations of intact FGF23 in patients receiving dialysis can increase 400 -fold from CKD stage 2-5, due not only to increased secretion from bone but also altered regulation and clearance $[7,30,31]$. The striking elevations and wide distribution of FGF23 among patients receiving dialysis may have masked associations of any of the FGF23 SNPs and serum concentrations of FGF23.

We did not find associations among SNPs in FGFR4 or $K L$ and cardiovascular events in the present study. Alpha$\mathrm{KL}$ is a required co-receptor for the kidney effects of FGF23 mediated through FGFR1, but not the cardiac effects mediated through FGFR4. Friedman et al. [13] found an association with 1-year mortality in patients on dialysis with the KL polymorphism rs 577912 in a cohort of 1,307 white and Asian patients on hemodialysis (RR 1.76; $95 \%$ CI 1.19-2.59; $p=0.003$ ) [12]. Interestingly, they found a more robust association in patients who were not receiving treatment for sHPT with calcitriol or its analogs, raising the possibility that treatment of sHPT with cinacalcet may have masked this association in our sample.

This study has several strengths. It is the largest genetic study in patients receiving dialysis to date with follow-up of up to 64 months. In addition, the cardiovascular endpoints were adjudicated, which is critically im- portant in studies of HF. In EVOLVE, nearly half of the investigator-designated HF events were adjudicated negative (Chertow, unpublished data) due to the difficulty in clinically diagnosing HF in patients receiving dialysis. Limitations include the modest sample size. Although large by dialysis clinical trial standards, we had fewer than 2,000 samples and only 747 primary composite cardiovascular endpoint events; therefore, we believe that we had insufficient power to detect other possible genetic associations or outcomes. Furthermore, patients in EVOLVE were enrolled because of moderate to severe secondary hyperparathyroidism despite therapy with phosphate binders and calcitriol or active vitamin $\mathrm{D}$ analogs; thus, these findings are not generalizable to all patients receiving hemodialysis or to other patients with ESKD, including patients treated with peritoneal dialysis, patients treated with nondialytic (conservative) therapy, or to kidney transplant recipients. Finally, there is strong LD between the 2 tested SNPs in FGF23 (chosen based upon prior literature $[11,15]$ ) and many, but not all, ungenotyped variants in that gene. Thus, the associated SNP might not be the functional one, and it is possible we missed an association with SNPs that are not in high LD with the tested SNPs. Finally, our genotype coverage of FGF23, FGFR4, or KL was not complete and thus possibly missed associations that were not in $\mathrm{LD}$ with the genotyped SNPs.

In conclusion, we report a novel association between rs 11063112 and cardiovascular mortality and HF events utilizing stored samples and adjudicated cardiovascular event data from EVOLVE. Although the mechanistic link between our findings of a significant association with rs 11063112 in FGF23 is not yet known, the finding is important in pointing out a target for future research. If validated, these results may help to recognize those patients receiving dialysis who are at increased risk for these events, or to identify persons who might be better suited for participation in clinical trials with FGF23-lowering therapies.

\section{Acknowledgments}

The authors dedicate this manuscript to the memory of Dr. Brian Decker, who passed away during the manuscript's final stage of editing but contributed to the planning and analyses of the study. Dr. Decker was a true scholar who had established a brilliant career dedicated to pharmacology and pharmacogenomics in CKD. His keen intellect and the resilience he exhibited during his illness will continue to inspire his co-authors, other colleagues, trainees, and students. 


\section{Ethics Statement}

The collection of DNA was under a separate consent during the primary trial, approved at each Institution. For the purposes of this use of the DNA samples, all data were deidentified and considered not subject to the Common Rule by the Indiana University Institutional Review Board.

\section{Disclosure Statement}

Dr. Moe reports grants from Chugai and Keryx, grants from $\mathrm{NIH}$ and Veterans Affairs, personal fees from Sanofi/Genzyme, personal fees from Amgen outside the submitted work. Dr. Cher- tow reports personal fees from Akebia, personal fees from AMAG, grants and personal fees from Amgen, personal fees and other from Ardelyx, personal fees from Astra Zeneca, personal fees from Baxter, personal fees from Gilead, personal fees from Keryx, other from Cricket Health, other from Durect, other from Outset Medical, other from Physiowave, other from PuraCath Medical, outside the submitted work. The other authors report no disclosures.

\section{Funding Sources}

This work was supported by NIH R01DK100306 to SMM and GMC. The EVOLVE trial was conducted and supported by AMGEN.

\section{References}

1 Moe S, Drueke T, Cunningham J, et al: Definition, evaluation, and classification of renal osteodystrophy: a position statement from kidney disease: improving global outcomes (KDIGO). Kidney Int 2006;69:19451953.

2 Faul C, Amaral AP, Oskouei B, et al: FGF23 induces left ventricular hypertrophy. J Clin Invest 2011;121:4393-4408.

3 Grabner A, Schramm K, Silswal N, et al: FGF23/FGFR4-mediated left ventricular hypertrophy is reversible. Sci Rep 16 2017;7: 1993.

4 Gutierrez OM, Januzzi JL, Isakova T, et al: Fibroblast growth factor 23 and left ventricular hypertrophy in chronic kidney disease. Circulation 19 2009;119:2545-2552.

5 Isakova T, Cai X, Lee J, et al: Longitudinal FGF23 Trajectories and Mortality in Patients with CKD. J Am Soc Nephrol 2018;29:579590.

6 Gutierrez OM, Mannstadt M, Isakova T, et al: Fibroblast growth factor 23 and mortality among patients undergoing hemodialysis. $\mathrm{N}$ Engl J Med 2008;359:584-592.

7 Moe SM, Chertow GM, Parfrey PS, et al: Cinacalcet, fibroblast growth factor-23, and cardiovascular disease in hemodialysis: the evaluation of cinacalcet $\mathrm{HCl}$ therapy to lower cardiovascular events (EVOLVE) trial. Circulation 2015;132:27-39.

8 Trial Investigators E, Chertow GM, Block GA, et al: Effect of cinacalcet on cardiovascular disease in patients undergoing dialysis. $\mathrm{N}$ Engl J Med 2012;367:2482-2494.

9 Chertow GM, Pupim LB, Block GA, et al: Evaluation of cinacalcet therapy to lower cardiovascular events (EVOLVE): rationale and design overview. Clin J Am Soc Nephrol 2007; 2:898-905.

10 Moe SM, Wetherill L, Decker BS, et al. Calcium-sensing receptor genotype and response to cinacalcet in patients undergoing hemodialysis. Clin J Am Soc Nephrol 2017;12:11281138
11 Pekkinen M, Laine CM, Makitie R, et al: FGF23 gene variation and its association with phosphate homeostasis and bone mineral density in Finnish children and adolescents. Bone 2015;71:124-130.

12 Zhu Q, Liu T: Fibroblast growth factor receptor 4 polymorphisms and coronary artery disease: a case control study. Mol Biol Rep 2012; 39:8679-8685.

13 Friedman DJ, Afkarian M, Tamez $\mathrm{H}$, et al: Klotho variants and chronic hemodialysis mortality. J Bone Miner Res 2009;24:18471855.

14 Ko GJ, Lee YM, Lee EA, et al: The association of Klotho gene polymorphism with the mortality of patients on maintenance dialysis. Clin Nephrol 2013;80:263-269.

15 Falcini F, Rigante D, Masi L, et al: Fibroblast growth factor 23 (FGF23) gene polymorphism in children with Kawasaki syndrome (KS) and susceptibility to cardiac abnormalities. Ital J Pediatr 2013;39:69.

16 Volkova N, McClellan W, Soucie JM, Schoolwerth A: Racial disparities in the prevalence of cardiovascular disease among incident end-stage renal disease patients. Nephrol Dial Transplant 2006;21:2202-2209.

17 Norris K, Mehrotra R, Nissenson AR: Racial differences in mortality and ESRD. Am J Kidney Dis 2008;52:205-208.

18 Wheeler DC, London GM, Parfrey PS, et al: Effects of cinacalcet on atherosclerotic and nonatherosclerotic cardiovascular events in patients receiving hemodialysis: the EValuation Of Cinacalcet $\mathrm{HCl}$ Therapy to Lower CardioVascular Events (EVOLVE) trial. J Am Heart Assoc 17 2014;3:e001363.

19 Eckardt KU, Gillespie IA, Kronenberg F, et al: High cardiovascular event rates occur within the first weeks of starting hemodialysis. Kidney Int 2015;88:1117-1125.

20 Foley RN, Parfrey PS, Harnett JD, et al: Clini$\mathrm{cal}$ and echocardiographic disease in patients starting end-stage renal disease therapy. Kidney Int 1995;47:186-192.
21 Zaslavsky LM, Pinotti AF, Gross JL: Diastolic dysfunction and mortality in diabetic patients on hemodialysis: a 4.25-year controlled prospective study. J Diabetes Complications 2005;19:194-200.

22 Kirkpantur A, Balci M, Gurbuz OA, et al: Serum fibroblast growth factor-23 (FGF-23) levels are independently associated with left ventricular mass and myocardial performance index in maintenance haemodialysis patients. Nephrol Dial Transplant 2011;26: 1346-1354.

23 Negishi K, Kobayashi M, Ochiai I, et al: Association between fibroblast growth factor 23 and left ventricular hypertrophy in maintenance hemodialysis patients. Comparison with B-type natriuretic peptide and cardiac troponin T. Circ J 2010;74:27342740.

24 Mitsnefes MM, Betoko A, Schneider MF, et al: FGF23 and left ventricular hypertrophy in children with CKD. Clin J Am Soc Nephrol 2018;13:45-52.

25 Di Marco GS, Reuter S, Kentrup D, et al: Treatment of established left ventricular hypertrophy with fibroblast growth factor receptor blockade in an animal model of CKD. Nephrol Dial Transplant 2014;29:20282035.

26 Grabner A, Amaral AP, Schramm K, et al: Activation of cardiac fibroblast growth factor receptor 4 causes left ventricular hypertrophy. Cell Metab 2015;22:1020-1032.

27 Leifheit-Nestler M, Grosse Siemer R, Flasbart $\mathrm{K}$, et al: Induction of cardiac FGF23/FGFR4 expression is associated with left ventricular hypertrophy in patients with chronic kidney disease. Nephrol Dial Transplant 2016;31: 1088-1099.

28 Touchberry CD, Green TM, Tchikrizov V, et al: FGF23 is a novel regulator of intracellular calcium and cardiac contractility in addition to cardiac hypertrophy. Am J Physiol Endocrinol Metab 2013;304:E863E873. 
29 Robinson-Cohen C, Bartz TM, Lai D, et al: Genetic variants associated with circulating fibroblast growth factor 23. J Am Soc Nephrol 2018;29:2583-2592.
30 Graciolli FG, Neves KR, Barreto F, et al: The complexity of chronic kidney disease-mineral and bone disorder across stages of chronic kidney disease. Kidney Int 2017;91:14361446.
31 Tagliabracci VS, Engel JL, Wiley SE, et al: Dynamic regulation of FGF23 by Fam20C phosphorylation, GalNAc-T3 glycosylation, and furin proteolysis. Proc Natl Acad Sci U S A 2014;111:5520-5525. 